\title{
ANÁLISIS DE LAS PROPIEDADES PSICOMÉTRICAS DE LA ESCALA DE CONDUCTAS VIOLENTAS EN DEPORTISTAS ADOLESCENTES
}

\author{
Félix Zurita Ortega \\ Silvia San Román Mata \\ Rubén Moreno Arrebola \\ Asunción Martínez Martínez \\ Gabriel González Varelo \\ Rosario Padial Ruz
}

https://doi.org/10.17060/ijodaep.2017.n1.v2.955

Fecha de Recepción: 12 Marzo 2017

Fecha de Admisión: 1 Abril 2017

\section{RESUMEN COMUNICACIÓN}

El presente estudio tiene como objeto determinar y analizar las propiedades psicométricas del instrumento de la escala de conducta violenta en la escuela propuesto por Little, Henrich, Jones y Hawley (2003) y adaptado al castellano por el grupo Lisis (Estévez, 2005) en una población de adolescentes deportistas. Los participantes fueron 1823 deportistas, con edades comprendidas entre los 13 y 17 de los cuales el $59,8 \%$ son masculinos y $40,2 \%$ femeninos, de ellos el $66,9 \%$ participan en deportes colectivos y el $33,1 \%$ en modalidades individuales, para el análisis estadístico se llevó a cabo un análisis factorial exploratorio y análisis de regresión mediante los programas estadísticos SPSS 22.0 y FACTOR Analysis 9.3.1 (Lorenzo-Seva y Ferrando, 2006), donde se exploran las propiedades métricas así como el efecto que tenían las horas de entrenamiento, años de práctica, género y modalidad, sobre las dimensiones del cuestionario escala de conducta violenta en la escuela de Estevez (2005). Los resultados, señalan un ajuste aceptable de la escala, que es aplicable a la población adolescente deportista, y demuestran la relación entre las variables personales y físico-deportivas con las dimensiones de la conducta violenta.

\section{INTRODUCCIÓN}

Actualmente y según señalan numerosas investigaciones las cifras de incidencia de violencia escolar, "Bullying", ha aumentado de una forma preocupante en todo el mundo (Cava, Musitu y Murgui, 2007; Cook, Williams, Guerra, Kim y Sadek, 2010; Povedano, Estévez, Martínez y Monreal, 2012; Zurita, Vilches, Cachón, Padial, Martínez y Castro, 2015). Los estudios de investigación realizados en España apuntan a que su incidencia va incrementándose paulatinamente.

Otra cuestión estrechamente relacionada con los comportamientos agresivos y perfiles psicoso- 


\section{ANÁLISIS DE LAS PROPIEDADES PSICOMÉTRICAS DE LA ESCALA DE CONDUCTAS VIOLENTAS EN DEPORTISTAS ADOLESCENTES}

ciales es la de la práctica de Actividad Física, son numerosos los autores que puntualizan la importancia que el deporte tiene para la adquisición de valores (Pelegrín, Martínez y Garcés de los Fayos, 2000; Pelegrín, 2004 o Collado, 2005); pero en otros estudios como los de Pelegrín (2004) y ChahínPinzon y Libia (2011) apreciaron en la relación entre agresividad y actividad física que el deporte arroja beneficios, tanto fisiológicos como psicológicos, favoreciendo el control de impulsos y por tanto los niveles de agresividad, pero Duda, Olson y Templin (1991); Duda y Huston (1995) y Gómez (2007), señalan que los deportistas jóvenes comienzan a competir, la derrota genera frustración, aumentando el intentar demostrar mejor habilidad que los demás, y estableciendo una relación entre el resultado y la agresión, trasladando esa competitividad a su vida social de una manera inconsciente.

De esta manera, en base a los factores psicosociales se puede generar éxito o fracaso según lo que consiga con la práctica de AF (Crocker, Sommers y Luthanen., 2002; Crocker, Brook, Niiya y Villacorta, 2006).

Se constata una carencia de test relacionados con la violencia en adolescentes en relación con la actividad física, este estudio tiene como objetivo determinar y analizar las propiedades psicométricas del instrumento de la escala de conducta violenta en la escuela y su aplicación al conjunto de adolescentes deportistas federados.

\section{MÉTODO}

\section{Participantes}

La muestra de este trabajo de investigación estuvo formada por 1823 adolescentes deportistas de entre 13 y 16 años ( $M=14.78$ años; $D T=1.123)$, divididos en $n=1090$ hombres (59.8\%) y $n=733$ mujeres $(40.2 \%)$. La muestra se obtuvo mediante un muestreo aleatorio simple en adolescentes. Se debe indicar que se detectaron 138 cuestionarios erróneos en su realización que fueron excluidos del estudio. La distribución de la muestra y los datos sociodemográficos se aprecian en la siguiente tabla:

Tabla 1.

Distribución de la muestra y datos sociodemográficos

\begin{tabular}{rcccc}
\hline Modalidad Deportiva & Individual & Colectivo & Horas Semana & TOTAL \\
\hline Masculino & $28.3 \%(\mathrm{~N}=308)$ & $71.7 \%(\mathrm{~N}=782)$ & $\mathrm{X}=4.20$ & $1090(100.0 \%)$ \\
\hline Femenino & $40.2 \%(\mathrm{~N}=295)$ & $59.8 \%(\mathrm{~N}=432)$ & $\mathrm{X}=3.33$ & $733(100.0 \%)$ \\
\hline
\end{tabular}

\section{Instrumentos}

Para la investigación se ha utilizado la Escala de Conductas Violentas en la Escuela propuesto por Little, Henrich, Jones y Hawley (2003) y adaptado al castellano por el grupo Lisis (Estévez, 2005), y está constituido por 25 preguntas agrupadas en seis dimensiones:

a) Agresión Manifiesta Pura: (Ítems $1+7+13+19$ ).

b) Agresión Manifiesta Reactiva: (Ítems $8+11+14+20$ ).

c) Agresión Manifiesta Instrumental: (Ítems 3+9+15+21+25).

d) Agresión Relacional Pura: (Ítems 4+10+16+22).

e) Agresión Relacional Reactiva: (Ítems $2+5+17+23$ ).

f) Agresión Relacional Instrumental: (Ítems $6+12+18+24$ ).

Los participantes responden en una escala entre 1 y 4 , donde el 1 es nunca y el 4 es siempre, y para determinar las dimensiones se establece el sumatorio de los ítems referenciados anteriormente. 


\section{Procedimiento}

Los cuestionarios se administraron en horario externo a las horas de clase y se aplicaron de manera grupal por los investigadores, preparados para tal efecto, después de hablar con los propios estudiantes. Se garantizó a los estudiantes el anonimato de la información recogida y aclarando que su utilización sería sólo con fines científicos.

\section{Análisis de los Datos}

En este estudio, las propiedades psicométricas (fiabilidad y análisis factorial exploratorio) se realizan mediante el paquete estadístico SPSS 22.0 para Windows y el Programa FACTOR Analysis 9.3.1 (Lorenzo-Seva y Ferrando, 2006). En primer lugar se emplea el SPSS 22.0., para analizar las propiedades métricas de cada ítem se emplearon los coeficientes descriptivos básicos. En segundo lugar se explora y utiliza el FACTOR, donde el diagnóstico de la bondad de ajuste es esencial para establecer la validez de la escala. La evaluación se ha basado en varios criterios como recomiendan Bentler (1990) y McDonald y Marsh (1990). Con la finalidad de verificar y determinar la consistencia interna del instrumento y de las distintas dimensiones se usó el coeficiente alpha de Cronbach.

\section{RESULTADOS}

En esta primera parte del análisis de los resultados se han calculado los valores descriptivos del estudio, y siguiendo los pasos recomendados por los expertos (Schmider, Ziegler, Danay, Beyer y Bühner, 2010) se ha tomado la decisión de no prescindir ningún dato, ya que todos presentan cifras inferiores a 2.00 en las pruebas de dispersión (asimetría y curtosis).

Tabla 2.

Descriptivos de los ítems de conducta violenta

\begin{tabular}{|c|c|c|c|c|c|c|}
\hline & Media & DT & Varianza & Asimetría & Curtosis & Rango \\
\hline V 01 & 1,35 & ,559 & ,313 &,- 529 &,- 894 & 3 \\
\hline V 02 & 1,65 & 837 &, 701 &,- 287 & $-1,026$ & 3 \\
\hline V 03 & 1,11 &, 384 &, 148 &, 377 & $-1,278$ & 3 \\
\hline V 04 & 1,13 &, 385 & ,148 &,- 724 &,- 491 & 3 \\
\hline V05 & 1,26 &, 531 & ,282 & $-1,456$ &, 981 & 3 \\
\hline V06 & 1,09 & ,322 & ,104 &,- 587 &,- 750 & 3 \\
\hline V 07 & 1,21 &, 507 &, 257 & $-1,606$ & 1,546 & 3 \\
\hline V 08 & 1,69 &, 897 &, 805 & $-1,938$ & 1,973 & 3 \\
\hline V 09 & 1,11 & ,407 & 166 & $-1,047$ &, 074 & 3 \\
\hline V 10 & 1,11 &, 370 &, 137 & $-1,547$ & 1,598 & 3 \\
\hline V 11 & 1,91 & 1,025 & 1,051 &,- 797 &,- 231 & 3 \\
\hline V 12 & 1,14 &, 407 &, 165 &,- 957 &,- 138 & 3 \\
\hline V 13 & 1,25 & ,499 & ,249 & $-1,168$ &, 229 & 3 \\
\hline V 14 & 1,28 &, 597 &, 357 & $-1,208$ &, 315 & 3 \\
\hline V 15 & 1,09 & 343 &, 118 &,- 924 &,- 112 & 3 \\
\hline V 16 & 1,25 &, 531 &, 282 &,- 793 &,- 183 & 3 \\
\hline V 17 & 1,69 &, 833 & ,694 &,- 555 &,- 989 & 3 \\
\hline V 18 & 1,18 & ,477 &, 227 &,- 587 &,- 887 & 3 \\
\hline V 19 & 1,14 & ,422 &, 178 & $-1,276$ &, 797 & 3 \\
\hline V 20 & 1,43 &, 731 &, 534 &, 511 & $-1,184$ & 3 \\
\hline $\mathrm{V} 21$ & 1,24 & ,477 &, 227 &,- 524 & $-1,098$ & 3 \\
\hline V 22 & 1,28 &, 557 &, 311 &,- 779 &,- 357 & 3 \\
\hline V 23 & 1,28 &, 540 & ,292 &,- 777 &,- 556 & 3 \\
\hline V 24 & 1,11 & ,349 & ,121 & $-1,004$ &, 129 & 3 \\
\hline V 25 & 1,15 & ,430 & ,185 & $-1,079$ & ,262 & 3 \\
\hline
\end{tabular}




\section{ANÁLISIS DE LAS PROPIEDADES PSICOMÉTRICAS DE LA ESCALA DE CONDUCTAS VIOLENTAS EN DEPORTISTAS ADOLESCENTES}

Seguidamente, mediante la utilización del programa FACTOR Analysis (Lorenzo-Seva y Ferrando, 2006) se han rotando seis factores. El estadístico de Bartlett, [13092.8 ( $\mathrm{df}=300 ; p=$ $0.000)$ ] y el test de Kaiser-Meyer-0lkin $(\mathrm{KMO})=0.91$, utilizados para probar si la muestra procede de poblaciones con la misma varianza y si presenta una buena adecuación muestral, indican un muy buen ajuste de los datos para ser sometidos al análisis factorial. Los seis factores extraídos explican el $54.9 \%$ de la varianza total, el CFI (índice de ajuste comparativo) fue de 0.96 , el índice de bondad de ajuste (GFI) fue de 0.99, el AGFI también obtuvo un 0.99 y la raíz cuadrática media de los residuales (RMSR) de 0.021. Todos estos datos nos indican un excelente ajuste para estos ítems.

En la siguiente tabla ha desaparecido la variable (V 13), por cargar en factores con un valor inferior a 0.300 , y la $\vee 18$ y $\vee 20$. La escala definitiva ha quedado formada por seis factores, el primero denominado agresión relacional instrumental formado por tres variables. El segundo factor está constituido por cuatro variables se relaciona con la agresión relacional reactiva; un tercer factor donde se agrupan tres variables coincide con la agresión manifiesta pura; un cuarto factor de agresividad relacional pura formado por cuatro variables; una quinta dimensión de agresión manifiesta instrumental formada por cinco variables; y un sexto factor de agresión manifiesta reactiva formado por tres variables.

Tabla 3.

Matriz Factorial Rotada

\begin{tabular}{|c|c|c|c|c|c|c|}
\hline & F1 & F2 & F3 & F4 & F5 & F6 \\
\hline V 01 & -0.094 & -0.006 & 0.409 & -0.014 & 0.281 & 0.070 \\
\hline V 02 & -0.012 & 0.711 & 0.009 & -0.170 & 0.012 & 0.113 \\
\hline V 03 & -0.037 & -0.083 & 0.059 & 0.061 & 0.640 & -0.026 \\
\hline V 04 & 0.104 & 0.003 & 0.015 & 0.539 & -0.036 & -0.054 \\
\hline V 05 & 0.012 & 0.541 & -0.023 & -0.210 & 0.043 & 0.251 \\
\hline V 06 & 0.675 & -0.019 & 0.048 & 0.137 & -0.083 & -0.191 \\
\hline V 07 & 0.023 & -0.026 & 0.569 & -0.078 & 0.308 & -0.157 \\
\hline V 08 & -0.054 & 0.011 & 0.227 & -0.048 & 0.068 & 0.506 \\
\hline V 09 & -0.031 & -0.014 & 0.123 & 0.129 & 0.452 & -0.045 \\
\hline V 10 & 0.136 & 0.108 & -0.054 & 0.252 & 0.082 & -0.018 \\
\hline V 11 & 0.114 & 0.025 & -0.009 & -0.187 & 0.125 & 0.557 \\
\hline V 12 & 0.400 & -0.050 & -0.085 & 0.180 & 0.099 & 0.032 \\
\hline V 13 & -0.060 & 0.182 & 0.018 & 0.211 & 0.242 & 0.108 \\
\hline V 14 & 0.027 & 0.034 & 0.168 & 0.069 & -0.159 & 0.902 \\
\hline V 15 & 0.043 & 0.011 & -0.005 & 0.171 & 0.599 & -0.133 \\
\hline V 16 & -0.029 & -0.079 & -0.106 & 0.481 & 0.139 & 0.274 \\
\hline V 17 & -0.024 & 0.525 & 0.094 & 0.331 & -0.211 & 0.323 \\
\hline V 18 & 0.146 & 0.038 & -0.003 & 0.512 & -0.082 & 0.037 \\
\hline V 19 & -0.043 & 0.076 & 0.618 & 0.092 & 0.012 & -0.038 \\
\hline V 20 & -0.010 & -0.053 & 0.663 & 0.204 & -0.036 & 0.049 \\
\hline V 21 & -0.022 & 0.115 & 0.025 & -0.172 & 0.555 & 0.158 \\
\hline V 22 & -0.129 & 0.056 & -0.091 & 0.632 & 0.015 & 0.039 \\
\hline V 23 & 0.039 & 0.791 & 0.048 & -0.087 & -0.149 & 0.031 \\
\hline V 24 & 0.538 & 0.122 & 0.005 & -0.007 & 0.122 & -0.135 \\
\hline V 25 & 0.078 & 0.006 & 0.066 & -0.115 & 0.652 & 0.026 \\
\hline
\end{tabular}

A continuación se muestra la configuración del cuestionario con un buen ajuste, el coeficiente de fiabilidad del cuestionario fue de 0.848 , y suprimidas las variables, por factores se obtuvo en todos ellos un alpha de Cronbach con valores $p>0.700$. 
Tabla 4.

Carga factorial de las dimensiones del Escala de Conductas Violentas

\begin{tabular}{|c|c|c|c|c|c|c|}
\hline Variables & F1 & F2 & F3 & F4 & F5 & F6 \\
\hline V 6 & 0.675 & & & & & \\
\hline V 12 & 0.400 & & & & & \\
\hline V 24 & 0.538 & & & & & \\
\hline V 02 & & 0.711 & & & & \\
\hline V 05 & & 0.541 & & & & \\
\hline V 17 & & 0.525 & & & & \\
\hline V 23 & & 0.791 & & & & \\
\hline V 01 & & & 0.409 & & & \\
\hline V 07 & & & 0.569 & & & \\
\hline V 19 & & & 0.618 & & & \\
\hline V 04 & & & & 0.539 & & \\
\hline V 10 & & & & 0.252 & & \\
\hline V 16 & & & & 0.481 & & \\
\hline V 22 & & & & 0.632 & & \\
\hline V 03 & & & & & 0.640 & \\
\hline V 09 & & & & & 0.452 & \\
\hline V 15 & & & & & 0.599 & \\
\hline V 21 & & & & & 0.555 & \\
\hline V 25 & & & & & 0.652 & \\
\hline V 08 & & & & & & 0.506 \\
\hline V 11 & & & & & & 0.557 \\
\hline V 14 & & & & & & 0.902 \\
\hline$\alpha=0.848$ & $\alpha=0.722$ & $\alpha=0.822$ & $\alpha=0.718$ & $\alpha=0.775$ & $\alpha=0.824$ & $\alpha=0.707$ \\
\hline
\end{tabular}

Como se observa en la tabla el alpha de Cronbach ha salido satisfactorio en su totalidad y en cada uno de los factores y dimensiones presentando valores $p>0.700$. Al aplicar el coeficiente de correlación de Pearson, se aprecia que casi todos los factores presentan correlación significativa en un $99 \%$, $(p<0.01)$, destacando sobretodo el factor 2 con el 5 ya que se encuentran con valor cercano al 0.700 , el resto se hallan con valores elevados. Como se puede apreciar todas las correlaciones son positivas y directas mostrando una significatividad al nivel de 0.01 .

Tabla 5.

Correlación de los factores

\begin{tabular}{lccccc}
\hline \multicolumn{7}{c}{ Factor 1 } & Factor 2 & Factor 3 & Factor 4 & Factor 5 & Factor 6 \\
\hline Factor 1 & 1 & & & & \\
\hline Factor 2 &, $349^{* *}$ & 1 & & & \\
\hline Factor 3 &, $445^{* *}$ &, $466^{* *}$ & 1 & & \\
\hline Factor 4 &, $420^{* *}$ &, $408^{* *}$ &, $401^{* *}$ & 1 & \\
\hline Factor 5 &, $269^{* *}$ &, $623^{* *}$ &, $343^{* *}$ &, $384^{* *}$ & 1 \\
\hline Factor 6 &, $277^{* *}$ &, $457^{* *}$ &, $347^{* *}$ &, $498^{* *}$ &, $439^{* *}$ \\
\hline$* *$ La correlación es significativa al nivel 0.01 (bilateral). & \\
\hline
\end{tabular}

\section{DISCUSIÓN}

Para establecer los comportamientos violentos en el contexto deportivo de los adolescentes se considera imprescindible disponer de cuestionarios que reporten datos fiables y reales de lo que se quiere medir, por tanto el principal objetivo de este trabajo ha sido analizar las propiedades psicométricas del cuestionario de Escala de Conductas Violentas, y observar su adaptación y aplicación a la población de adolescentes deportistas de Granada, de esta manera los resultados obtenidos han 


\section{ANÁLISIS DE LAS PROPIEDADES PSICOMÉTRICAS DE LA ESCALA DE CONDUCTAS VIOLENTAS EN DEPORTISTAS ADOLESCENTES}

sido satisfactorios en cuanto a los coeficientes alpha de Cronbach, tanto en la totalidad de la escala como para los seis factores, mostrando que es un instrumento válido y fiable.

Los resultados se mantienen estables en todas las dimensiones, cargando los factores de una forma coherente aunque hay tres variables que se pierden, como ya se ha citado los datos obtenidos apoyan la teoría de conocer los comportamientos violentos en deportistas federados, presentando unos índices de ajuste aceptable y con una buena consistencia interna y estabilidad temporal, presentando valores que superan el 0.700 , obteniendo una adecuada fiabilidad. También se debe señalar que los datos obtenidos sugieren algunas cuestiones que deberían ser atendidas en próximos trabajos.

En este trabajo se encontró una alta correlación entre todas las dimensiones, por lo que se debe considerar la importancia del estudio y de los resultados obtenidos apoyando los resultados obtenidos por Zurita, Vilches, Padial, Pérez y Martínez (2015a) y Zurita et al. (2015b), quienes encontraron relaciones recurrentes entre la violencia y el deporte en adolescentes.

Los resultados obtenidos en este estudio, permiten concluir que el Cuestionario de Conductas Violentas cumple las especificaciones técnicas de validez y confiabilidad para ser utilizado en el contexto de los adolescentes deportistas, si bien resultaría relevante investigar la validez de criterio del instrumento al confrontarlo con otro de iguales, y podría utilizarse para establecer las posibles diferencias con otras variables (p. ej., género, campus, curso,....).

\section{REFERENCIAS}

Bentler, P. M. (1990). Comparative fit indexes in structural models. Psychological Bulletin, 107, 238246.

Cava, M.J., Musitu, G. y Murgui, S. (2007). Individual and social risk factors related to overt victimization in a sample of Spanish adolescents. Psychological Reports, 101, 275-290.

Chahín-Pinzón N. y Libia B. (2011). Actividad física en adolescentes y su relación con agresividad, impulsividad, Internet y videojuegos. Psychologia: Avances de la Disciplina, 5(1), 9-23.

Collado, D. (2005). Transmisión y adquisición de valores a través de un programa de Educación Física basado en el juego motor, en un grupo de alumnos y alumnas de Primero de la ESO. Tesis Doctoral. Universidad de Granada.

Cook, C., Williams, K., Guerra, N., Kim, T. y Sadek, S. (2010). Predictors of Bullying and Victimization in Childhood and Adolescence: A Meta-analytic Investigation. School Psychology Quarterly, 25(2), 65- 83.

Crocker J, Brook AT, Niiya Y y Villacorta M (2006). The Pursuit of Self-esteem: Contingencies of selfworth and self-regulation. Journal of Personality, 74, 1749-1771.

Crocker J., Sommers S.R. y Luhtanen, R.K. (2002). Hopes dashed and dreams fulfilled: Contingencies of self-worth and graduate school admissions. Personality and Social Psychology Bulletin, 28, 1275-1286.

Duda ,J.L., Olson, L.K. y Templin, T.J. (1991). The relation of task and ego orientations to sportsmanship attitudes and the perceived legitimacy of injurius acts. Research Quaterly for Exercise and Sport. 62, 79-87

Duda, J.L. y Huston, L. (1995). The relationship of goal orientation and degree of competitive sport participation to the endorsement of aggressive acts in American football. IXth Europen Congresson Sport Psychology, 665-662.

Estévez, E. (2005). Violencia, Victimización y Rechazo Escolar en la Adolescencia. Universitat de Valencia: Servei de publicacions.

Gómez, A. (2007). La violencia en el deporte: un análisis desde la psicología social. Revista de Psicología Social, 22(1), 63-87. 
Little, T. D., Henrich, C. C., Jones, S. M. y Hawley, P. H. (2003). Disentangling the "whys" from the "whats" of aggressive behaviour. International Journal of Behavioral Development, 27, 122-133.

Lorenzo-Seva, U. y Ferrando, P. J. (2006). FACTOR: A computer program to fit the exploratory factor analysis model. Behavioral Research Methods, Instruments and Computers, 38(1), 88-91.

McDonald, R. P. y Marsh, H. W. (1990). Choosing a multivariate model: noncentrality and goodness of fit. Psychological Bulletin, 107, 247-255.

Pelegrín, A. (2004). El comportamiento agresivo y violento: Factores de riesgo y protección como mediadores de inadaptaciones y adaptaciones en la socialización del niño y el adolescente. Tesis Doctoral: Universidad de Murcia.

Pelegrín, A., Martínez, F. y Garcés de los Fayos E.J. (2000). La actividad física y el deporte como factores controladores de conductas antisociales y delictivas. II Congreso Iberoamericano de Psicología del Deporte. Huelva.

Povedano, A., Estévez, E., Martínez, B. y Monreal, M.C. (2012). Un perfil psicosocial de adolescentes agresores y victimas en la escuela: análisis de las diferencias de género. Revista de Psicología Social, 27(2), 169-182.

Schmider, E., Ziegler, M., Danay, E., Beyer, L., y Bühner, M. (2010). Is it really robust? Reinvestigating the robustness of ANOVA against violations of the normal distribution assumption. Meythodology, 6, 147-151.

Zurita, F., Vilches, J. M., Cachón, J., Padial, R., Martínez, A., y Castro, M. (2015b). Violencia escolar en adolescentes: un análisis en función de la actividad física y lugar de residencia habitual. Universitas Psychologica, 14(2), 743-754.

Zurita, F., Vilches, J. M., Padial, R., Pérez, A., y Martínez, A. (2015a). Conductas agresivas y de Bullying desde la perspectiva de actividad física, lugar de residencia y género en adolescentes de Granada. Revista Complutense de Educación, 26(3), 527-542. 
